\title{
Patient adherence and preference considerations in managing cardiovascular risk: focus on single pill and amlodipine/atorvastatin fixed combination
}

\author{
Farhan Aslam \\ Attiya Haque \\ Veronica Lee \\ JoAnne Foody \\ Division of Cardiovascular Medicine, \\ Brigham and Women's Hospital, \\ Boston, MA, USA
}

\begin{abstract}
Cardiovascular disease (CVD) accounts for in excess of 930,000 deaths in the United States each year. Risk factors for CVD often co-exist. Studies estimate that over half of the hypertensive population also has dyslipidemia. Observational data suggest that fewer than $10 \%$ of patients attain recommended therapeutic targets for both conditions. A variety of patient, regimen and system characteristics have been associated with the risk for non-adherence. Polypharmacy and complex drug regimens are associated with poor patient adherence and thus the use of fixed-dose combination therapies, may improve adherence by reducing the pill burden. The fixed-dose combination of amlodipine/atorvastatin offers a convenient and effective approach to manage two important CVD risk factors. The combination of amlodipine/atorvastatin has a synergistic effect. The half-life of both agents facilitates once-daily dosing and both can be administered at any time of the day with or without food. Amlodipine/atorvastatin combined pill can be used to initiate both agents or patients can be switched directly from single-agent therapy with one or both agents. The convenience of single-pill amlodipine/atorvastatin has the potential to improve patient adherence and the management of cardiovascular risk in selected patients, thereby improving clinical outcomes.
\end{abstract}

Keywords: amlodipine/atorvastatin, cardiovascular disease, drug combination, adherence, poly-pharmacy

\section{Introduction}

Cardiovascular disease (CVD) accounts for in excess of 930,000 deaths and US $\$ 350$ billion in direct medication costs and loss of productivity in the United States each year. ${ }^{1}$ Risk factors for CVD often co-exist. For example, in a retrospective study among the general veteran population (mainly males) in the United States, $30.7 \%$ had both hypertension (HTN) and dyslipidemia (DL). ${ }^{2}$ Clinical studies have demonstrated a link between HTN and other metabolically linked risk factors (including metabolic $\mathrm{X}$ syndrome $)^{3}$ and various studies estimate that over half of the hypertensive population also has DL. ${ }^{4}$ It is also clear that CVD risk factors tend to increase the total or absolute CVD risk in a multiplicative rather than additive manner. ${ }^{5}$ Thus individual treatment strategies should be based on absolute CVD risks, determined by synergistic effect of all cardiovascular risk factors present, rather than on individual risk factors. ${ }^{6}$ Thus in hypertensive patients at high cardiovascular risk, the strategy is to include two or more antihypertensive $(\mathrm{AH})$ agents for blood pressure control and adding concomitant lipid lowering, 3-hydroxy-3-methylglutaryl co enzyme A reductase inhibitor (HMG-Co-A R or statin) therapy for CVD risk reduction. ${ }^{7,8}$

Although the importance of treating HTN and DL is well established in treatment guidelines, the current rate of control of these risk factors is unsatisfactory. ${ }^{9}$ United States epidemiological data have indicated that less than $5 \%$ of patients with 
concomitant HTN and DL were at goal for both conditions. ${ }^{10}$ The reasons for this are not clear, but may include failure to treat adequately and poor patient adherence. ${ }^{11}$ Poly-pharmacy and complex drug regimens are associated with poor patient adherence and thus the use of fixed-dose combination therapies, may improve adherence by reducing the pill burden. ${ }^{12}$ Indeed, recent data have shown that fixed-dose combination therapies improve patient adherence compared with the coadministration of single-agent formulation ${ }^{13}$ and that good adherence to drug therapy is associated with positive health outcomes. $^{14}$

\section{Definition}

Adherence Is defined as behavior that follows the recommendations of a health care provider or degree of prescription filling in a given interval.

Compliance is defined as the extent to which a person follows a doctor's order.

\section{Determinants of adherence}

A variety of patient, regimen and system characteristics have been associated with the risk for non-adherence (Table 1). Some patient characteristics include age, sex, race and presence of chronic diseases, like diabetes and depression. In one study there was significant disparity in persistence among Black and other non-White races which is of particular concern because Black and Mexican Americans have a higher prevalence of CVD than Whites. ${ }^{15}$ Older patients are 1.03 times as likely to be adherent compared with younger patients, while males were 1.42 times more likely to be adherent compared with females. ${ }^{16}$

Table I Determinants of non-adherence and poor persistence

I. Patient characteristics:
Female
Age under 45 years
Age over 75 years
Low socio-economic status
Non-White
Comorbidity: dementia, depression, myocardial infarction after statin
or antihypertensive use
Asymptomatic/feeling in good health
2. Regimen characteristics
Multiple daily dosing
Multiple drug regimens
3. System characteristics
Long waiting time
Lack of insurance
Medication cost

A history of stroke, congestive heart failure and diabetes predict better persistence. By contrast those who had myocardial infarction after starting AH/DL therapy were significantly less likely to continue with their medication following the event. ${ }^{17}$ Patients treated for depression were less likely to persist in AH/DL medication use, suggesting that depressive symptoms correlate with poor persistence with medication use. ${ }^{18}$

Adherence declines most rapidly during the first 6 months of concomitant AH and DL therapy, suggesting the importance of early interventions to maintain or improve adherence. ${ }^{19}$ Similarly, adherence is best when AH and DL therapies are initiated on or about the same time, suggesting benefit from concomitant initiation of therapy to treat these two CVD risk factors.

The number of other medications that a patient is already taking is strongly and inversely associated with adherence with concomitant therapy. ${ }^{20}$ Cost pressure also has a substantial influence on the prescription-taking attitude of patients, especially older patients. They may simply not fill the prescription, skip doses or take smaller doses to make the prescription last longer. ${ }^{21}$ Further, patients with more outpatient visits during the baseline period are more adherent than patients with fewer visits. Patients who had undergone a cardiovascular procedure or who had been hospitalized were more adherent, but patients who had at least one emergency department visit were less likely to be adherent than those who had not had an emergency visit. Patients who filled their medication prescription via mail order were more likely to be adherent than those who used retail pharmacy stores. ${ }^{22}$

\section{Need for adherence to modify cardiovascular risk factors}

Current guidelines for the management of HTN and DL have focused on the need to set blood pressure and lipid targets dependent on a patient's overall level of cardiovascular risk. ${ }^{23,24}$ However, observational data suggest that fewer than $10 \%$ of patients attain recommended therapeutic targets for both conditions. ${ }^{25}$ In clinical practice, long-term adherence and persistence with prescribed drug therapy are poor. Of all the written prescriptions, $14 \%$ are never filled and another $13 \%$ are filled but never taken. ${ }^{26}$ Among patients who actually initiate therapy with HMG-Co-A reductase inhibitor or statin, observational studies have reported 1-year discontinuation rates of $15 \%$ to $60 \%$ depending on the patient population and practice settings. ${ }^{27,28}$ Similar trends have been observed with AH medications. During the first year of AH therapy, it is noted that the average patient had filled AH prescription less 
than $50 \%$ of the time and 1 in 5 patients exhibit compliance sufficient to obtain the therapeutic benefits observed in clinical trials. $^{29}$

Patients with lower adherence are also responsible for substantially greater health care costs than are patients with good adherence. In one study, even accounting for savings in drug costs, patients who took less than $20 \%$ of their lipid lowering medications had more than US $\$ 3,000$ greater yearly health care costs than patients with $80 \%$ adherence. ${ }^{30}$

The under-recognition of medication non-adherence can have adverse consequences. ${ }^{31}$ For example, patients may have poor blood pressure control related to medication non-adherence. However, the clinician may attribute poor blood pressure control to therapeutic ineffectiveness and may increase dose of current medication or add more medications to the regimen.

On the other hand, lack of patient adherence to multiple therapies is common and is associated with poor clinical outcomes. ${ }^{32}$ As a result, concerns that increasing a patient's bill burden will decrease adherence may discourage physicians from adding further medication to a patients' existing regimen, despite potential therapeutic benefits. ${ }^{33,34}$ Thus better patient adherence is a cornerstone for therapeutic success and achievement of target goals in order to modify risk factors and decrease morbidity associated with CVD.

Therefore, medication non-adherence should be routinely assessed by the physician. Once identified, clinicians should explore the reasons for the non-adherence and implement measures to improve adherence - the most simple of which is simplifying therapeutic regimens.

\section{Interventions to improve adherence; simplifying the drug regimen}

There are a number of interventions to improve patient adherence. One of the simplest ways to improve adherence is to simplify the drug regimen by adding combination therapy. Compared with single-agent therapy, combination therapy is associated with increased compliance, greater response and lower cost (Table 2). A study within a large managed care population $(n=8406)$ showed that patients who initiated both AH and DL therapies within 30 days of each other were more likely to be adherent to both drugs over time. ${ }^{35}$ After 4 months, patients who were prescribed AH and DL therapy together were $5 \%$ to $24 \%$ more adherent to therapy than those who were prescribed AH and DL therapy separately.

Several studies have explored the role of single-pill combinations in improving adherence rates. In particular, the single-pill combination of atorvastatin and amlodipine
Table 2 Advantage of fixed-dose combination therapy

Increased compliance, simplified titration and convenience of use Additive or synergistic effects

Enhancing effects in specific populations

Attenuation in adverse events

Improved overall results

Greater response

Lower cost

Availability in a variety of dosing combinations, making it easy to escalate dose of one medicine while keeping same dose for other medicine (Caduet)

(Caduet $^{\circledR}$, Pfizer, Inc) has provided a unique opportunity to explore the issue of adherence in individuals with both hypertension and dyslipidemia. ${ }^{36}$ Amlodipine besylate is a dihydropyidine calcium channel blocker (CCB) used for treatment of hypertension and both vaso-spastic and chronic stable angina. ${ }^{37}$ Atorvastatin is a $\mathrm{HMG}-\mathrm{Co}-\mathrm{A}$ reductase inhibitor that has been shown to be an effective and safe treatment for dyslipidemia and is also effective in reducing the rate of coronary events and revascularization procedure in patients with multiple cardiovascular risk factors. ${ }^{38,39}$

The combination of amlodipine and atorvastatin has a synergistic effect. Amlodipine in clinical studies has demonstrated some antiatherosclerotic properties. ${ }^{40}$ A potential synergistic and dose-dependent increase in nitric oxide (NO) has been observed with this combination (NO is a potent arterial vasodilator). ${ }^{41}$ Additional synergistic effects observed with this combination include improved arterial wall compliance $^{42}$ and decrease in insulin resistance. ${ }^{43}$ The pharmacokinetic and pharmacodynamic properties of amlodipine and atorvastatin make them well suited for combination in a single pill to manage cardiovascular risk. ${ }^{44,45}$ The half-life of both agents facilitates once-daily dosing and both can be administrated at any time of the day with or without food. Another advantage of this combination is its availability in a variety of combination dosage (eg, 2.5/10, 2.5/20, 2.5/40, $5 / 10,5 / 20$ ), thus making it easier for the clinician to escalate the dose of one agent to achieve target therapeutic blood pressure and cholesterol levels.

The co-administration of amlodipine and atorvastatin has been demonstrated to be safe and effective at lowering both blood pressure and low density lipoprotein cholesterol (LDL-C) levels in patients with concomitant HTN and DL.

Amlodipine/atorvastatin combined pill can be used to initiate both agents or patients can be switched directly from single-agent therapy with one or both agents. ${ }^{46}$

The GEMINI study was a 14-week open-label study designed to simulate real world clinical practice. ${ }^{47}$ 
Table 3 Results of trials of amlodipine and atorvastatin combination

\begin{tabular}{lllllll}
\hline Study & $\begin{array}{l}\text { No of } \\
\text { patients }\end{array}$ & $\begin{array}{l}\text { Baseline value } \\
\text { SPB/DBP } \\
(\mathbf{m m H g})\end{array}$ & $\begin{array}{l}\text { Baseline value } \\
\text { LDL-C } \\
(\mathbf{m g} / \mathbf{d L})\end{array}$ & $\begin{array}{l}\text { Patients achieving } \\
\text { BP and LDL-C } \\
\text { goals (\%) }\end{array}$ & $\begin{array}{l}\text { Patients achieving } \\
\text { BP goals (\%) }\end{array}$ & $\begin{array}{l}\text { Patients achieving } \\
\text { LDL-C goals (\%) }\end{array}$ \\
\hline CAPABLE & 499 & $147 / 91$ & 142.2 & 48.3 & 56.8 & 73.7 \\
GEMINI & 1220 & $146 / 88$ & 153 & 57.7 & 65.5 & 74.7 \\
GEMINI-AALA & 1649 & $146 / 88$ & 131.3 & 55.2 & 61.3 & 87.1 \\
JEWEL I & 1138 & $152 / 90$ & 193 & 62.9 & 66.8 & 90.7 \\
JEWEL II & 1107 & $152 / 90$ & 193 & 50.6 & 65.7 & 73.1 \\
\hline
\end{tabular}

Abbreviations: SBP, systolic blood pressure; DBP, diastolic blood pressure; LDL-C, low density lipoprotein cholesterol.

Almost $60 \%$ of all patients and more than $75 \%$ of patients without CVD or CVD risk equivalents met target levels for both blood pressure and LDL-C after a 14-week period and 3 titration visits.

The JEWEL Program consisted of 2 similar 16-week studies (JEWEL I in UK and Canada, JEWEL II in Europe) involving a total of 2219 patients (intent to treat population). ${ }^{48}$ Eight fixed doses of amlodipine/atorvastatin were administered to establish country-specific target reduction in blood pressure and LDL-C. At end point, the percentage of patients who achieved both their target blood pressure and LDL-C were $62.9 \%$ in JEWEL I and 50.6\% in JEWEL II, respectively. ${ }^{49}$

Similar results were noted in RESPOND ${ }^{50}$ (conducted in the US), GEMINI-AALA ${ }^{51}$ (conducted in Asia Pacific, Middle East and Africa) and CAPABLE ${ }^{52}$ (conducted in an African-American population in the US) trials (Table 3).

In observational studies, similar results have been observed. In one study, 5 cohorts representing 4703 patients were observed depending on their CCB and statin therapy ${ }^{53}$ (1. amlodipine/atorvastatin [single pill]; 2. amlodipine + atorvastatin; 3. amlodipine + other statin [not including atorvastatin]; 4. atorvastatin + other $\mathrm{CCB}$ [not including amlodipine]; 5. other $\mathrm{CCB}+$ other statin [not including atorvastatin or amlodipine]).

Adherence was measured during a 6-month post-baseline period as proportion of days covered (PDC), based on the days that both antihypertensive and lipid-lowering drugs were supplied. Patients were considered to be adherent if their PDC was $\geq 80 \%$. The probability of achieving adherence using single-pill amlodipine/atorvastatin was 1.95-times greater than with the parent compounds amlodipine and atorvastatin administered separately ( $\mathrm{p}<0.0001$ ), and ranged from 2.06to 3.10-times greater than for any of the other CCB and statin regimens studied $(\mathrm{p}<0.0001)$. For the additional 1-year analysis, patients receiving amlodipine/atorvastatin were 2.41- to 3.50-times more likely to achieve adherence than patients on a 2-pill CCB and statin regimen. Furthermore, patients taking single-pill amlodipine/atorvastatin were less likely to discontinue therapies compared with those taking the parent compounds or other CCB and statin combinations.

\section{Conclusion}

The overall goal in the prevention of CVD is to improve the multivariate risk profiles, rather than treating single risk factors. Given the significant burden of non-adherence in society, better strategies are required to improve medication adherence so that all patients may benefit from evidence-based therapies. A combination of patient-focused, physicianfocused and system-focused interventions work best.

The fixed-dose combination of amlodipine/atorvastatin offers a convenient and effective approach to manage two important CVD risk factors. The combination is generally well tolerated and bioequivalent to amlodipine and atorvastatin given alone. The convenience of single-pill amlodipine/ atorvastatin has the potential to improve patient adherence and the management of cardiovascular risk in selected patients, thereby improving clinical outcomes.

\section{Disclosures}

Dr Foody is a consultant for Merck \& Co., Merck/ Schering-Plough, Pfizer and Sanofi-Aventis.

\section{References}

1. American Heart Association. Heart Disease and Stroke Statistics: 2004 Update. Dallas, Tex: American Heart Association; 2003.

2. Johnson ML, Pietz K, Battleman DS, et al. Prevalence of comorbid hypertension and dyslipidemia and associated cardiovascular disease. Am J Manag Care. 2004;10:926-932.

3. Kannel WB. Risk stratification in hypertension: new insights from the Framingham Study. Am J Hypertension. 2000;13:3S-10S.

4. Cowie MR. Simultaneous treatment of hypertension and dyslipidaemia may help to reduce overall cardiovascular risk: focus on amlodipine/ atorvastatin single-pilltherapy. Int J Clin Pract. 2005;59(7):839-846.

5. Rosendorff C, Black HR, Cannon CP, et al. Treatment of hypertension in the prevention and management of ischemic heart disease. Circulation. 2007;115:2761-2788. 
6. Jackson R, Lawes CMM, Bennertt DA, et al. Treatment with drugs to lower blood pressure and blood cholesterol based on an individual's absolute cardiovascularrisk. Lancet. 2005;365:434-441.

7. Messerli FH, Williams B, Ritz E. Essential hypertension. Lancet. 2007;370:591-603.

8. The Task Force for the Management of Arterial Hypertension of the European Society of Hypertension (ESH) and of the European Society of Cardiology (ESC). Guidelines for the management of arterial hypertension. Eur Heart J. 2007;28:1462-1536.

9. Kostis BK. The importance of managing hypertension and dyslipidemia to decrease cardiovascular disease. Cardiovasc Drugs Ther. 2007;21:297-309.

10. Battleman DS, Peterson ED. Estimated prevalence of comorbid hypertension and dyslipidemia and therapeutic goal attainmen among US adults in 2000, utilizing data from the National Health and Nutrition Examination Survey [abstract]. J Manag Care Pharm. 2004;10(2):186.

11. Heart Protection Study Collaborative Group. MRC/BHF health protection study of cholesterol lowering with simvastatin in 20536 high-risk individuals: a randomized placebo-controlled trial. Lancet. 2002;360:7-22.

12. Bangalore S, Kamalakkannan G, Parkar S, et al. Fixed-dose combinations improve medication compliance: a meta-analysis. $\mathrm{Am} \mathrm{J} \mathrm{Med}$ 2007;120:713-719.

13. Dezii CM. A retrospective study of persistence with single-pill combination therapy vs concurrent two-pill therapy in patients with hypertension. Manag Care. 2000;9 Suppl:2-6.

14. Simpson SH, Eurich DT, Majumdar SR, et al. A meta-analysis of the association between adherence to drug therapy and mortality. $B M J$ 2006;333(15):E1-E6.

15. Bosworth HB, Dudley T, Olsen MK, et al. Racial differences in blood pressure control: potential explanatory factors. Am J Med. 2006;119(70): e9-e15.

16. Benner JS, Glynn RJ, Mogun H, et al. Long-term persistence in use of statin therapy in elderly patients. JAMA. 2002;288:455-461.

17. Gerbino PP, Shoheiber O. Adherence patterns among patients treated with fixed dose combination versus separate antihypertensive agents. Am J Health Syst Pharm. 2007;47:1039-1048.

18. Wang PJ, Bohn RL, Knight E, et al. Noncompliance with antihypertensive medications: the impact of depressive symptoms and psychosocial factors. J Gen Intern Med. 2002;17(7):504-511.

19. Schwartz JS, Mclaughlin T, Griffis D, et al. Adherence to chronic therapy among patients treated for hypertension, dyslipidemia or both [abstract]. J Am Coll Cardiol. 2003;41 suppl A:526A.

20. Avorn J, Monette J, Lacour A, et al. Persistence of use of lipid-lowering medications: a cross-national study. JAMA. 1998;279:1458-1462.

21. Safran DG, Neuman P, Schoen C, et al. Prescription drug coverage and seniors: findings from a 2003 national survey. Where do things stand on the eve of implementing the new Medicare Part D benefit? Data Watch. 2005;DOI 10.1377/hlthaff.W5.152.W5-152-W5-166.

22. Schultz JS, O'Donnell JC, McDonough KL, et al. Determinants of compliance with statin therapy and low-density lipoprotein cholesterol goal attainment in a managed care population. Am J Manag Care 2005;11:306-312.

23. The Seventh Report of the Joint National Committee on Prevention, Detection, Evaluation, and Treatment of High Blood Pressure: The JNC7 Report. JAMA. 2003;289:2560-2572.

24. National Cholesterol Education Program (NCEP) Expert Panel on Detection, Evaluation, and Treatment of High Blood Cholesterol in Adults (Adult Treatment Panel III) Third Report of the National Cholesterol Education Program (NCEP) Expert Panel on Detection, Evaluation and Treatment of High Blood Cholesterol in adults (Adult treatment panel III) final report. Circulation. 2002;106:3143-3421.

25. Pettitt D, Karter AJ, Peng TY, et al. The impact of concurrent dyslipidemia and diabetes on hypertension management and goal attainment. Poster presented at: Annual meeting of the Society of General Internal Medicine; April 30-May 3, 2003, Vancouver, Canada.
26. Berg JS, Dischler J, Wagner DJ, et al. Medication compliance: a healthcare problem. Ann Pharmacother. 1993;27 suppl:S1-S24.

27. Andrade SE, Walker AM, Gottlieb LK, et al. Discontinuation of antihyperlipidemic drugs: do rates reported in clinical trials reflect rates in primary care settings? N Engl J Med. 1995;332:1125-1131.

28. Simons LA, Levis G, Simons J, et al. Apparent discontinuation rates in patients prescribed lipid-lowering drugs. Med J Aust. 1996; 164:208-211.

29. Monane M, Bohn RL, Gurwitz JH, et al. The effects of initial drug choice and comorbidity on antihypertensive therapy compliance: results from a population-based study in the elderly. Am J Hypertens. 1997; 10:697-704.

30. McCombs JS, Nichol MB, Newman CM, et al. The costs of interrupting antihypertensive drug therapy in a Medicaid population. Med Care. 1994;32: 214-226.

31. Kogut SJ, Andrade SE, Willey C, et al. Non-adherence as a predictor of anti-diabetic drug therapy intensification. Pharmacoepidemiol Drug Saf. 2004;13:591-598.

32. Blackburn DF, Dobson RT, Blackburn JL, et al. Adherence to statins, beta-blockers and angiotensin-converting enzyme inhibitors following a first cardio vascular event: a retrospective cohort study. Can J Cardiol. 2005;21:485-488

33. Ho PM, Rumsfeld JS, Masoudi FA, et al. Effect of medication nonadherence on hospitalization and mortality among patients with diabetes mellitus. Arch Intern Med. 2006;166:1836-1841.

34. Kulkarni SP, Alexander KP, Lytle B, et al. Long-term adherence with cardiovascular drug regimens. Am Heart J. 2006;151:185-191.

35. Chapman RH, Benner JS, Petrilla AA, et al. Predictors of adherence with antihypertensive and lipid-lowering therapy. Arch Intern Med. 2005;165:1147-1152.

36. McKeage KM, Siddiqui AA. Amlodipine/atorvastatin fixed-dose combination. a review of its use in the prevention of cardiovascular disease and in the treatment of hypertension and dyslipidemia. Am J Cardiovasc Drugs. 2008;8(1):51-67.

37. Kass RS, Arena JP, DiManno D. Block of heart calcium channels by amlodipine: influence of drug charge on blocking activity. J Cardiovasc Pharmacol. 1988;12 suppl 7:S45-49.

38. Lipitor (atorvastatin calcium) Tablets. US Prescribing Information. New York, NY: Pfizer Inc; 2004.

39. Newman CB, Palmer G, Silbershatz H, et al. Safety of atorvastatin derived from analysis of 44 completed trials in 9416 patients. Am J Cardiol. 2003;92:670-676.

40. Jukema JW, van der Hoorn JW. Amlodipine and atorvastatin in atherosclerosis: a review of the potential of combination therapy. Expert Opin Pharmacother. 2004;5(2):459-468.

41. Mason RP. A rational for combined therapy with a calcium channel blocker and a statin: evaluation of basic and clinical evidence. Curr Drug Targets Cardiovasc Haematol Disord. 2005;5(6):489-501.

42. Leibovitz E, Beniashvili M, Zimlichman R, et al. Treatment with amlodipine and atorvastatin have additive effect in improvement of arterial compliance in hypertensive hyperlipidemic patients. Am J Hypertens. 2003;16(9 Pt 1):715-718.

43. Fogari R, Preti P, Zoppi A, et al. Effects of amlodipine-atorvastatin combination on inflammation markers and insulin sensitivity in normocholesterolemic obese hypertensive patients. Eur J Clin Pharmacol. 2006;62:817-822.

44. Malhotra HS, Goa KL. Atorvastatin: an updated review of its pharmacological properties and use in dyslipidaemia. Drugs. 2001; 61(12):1835-1881.

45. Murdoch D, Heel RC. Amlodipine: a review of its pharmacological and pharmacookinetic properties, and therapeutic use in cardiovascular disease. Drugs. 1991;41(3):478-505.

46. Pfizer Labs. Caduet (amlodipine besylate/atorvastatin calcium) tablets: description [online]. Available from URL: http://www.caduet.com.

47. Blank R, LaSalle J, Reeves R, et al. Single pill therapy in the treatment of concomitant hypertension and dyslipidemia (the amlodpine/atorvastatin Gemini Study). J Clin Hypertens. 2005;7(5):264-273. 
48. Hobbs FD, Gensini G, Mancini GB, et al. Can combining different risk interventions into a single formulation contribute to improved cardiovascular disease risk reduction? Rationale and design for an international, open-label program to assess the effectiveness of a single pill (amlodipine/ atorvastatin) to attain recommended target levels for blood pressure and lipids (The JEWEL Program). Int J Cardiol. 2006;110:242-250.

49. Feldman R. Multiple risk intervention with a single-pill combination (amlodipine/atorvastatin) helps patients to attain recommended target levels for blood pressure and lipids (The JEWEL Program) [abstract no. P-129B]. J Clin Hypertens. 2006;8(6):457.

50. Preston RA, Harvey $\mathrm{P}$, Herfert $\mathrm{O}$, et al. A randomized, placebocontrolled trial to evaluate the efficacy, safety, and pharmacodynamic interaction of co-administered amlodipine and atorvastatin in 1660 patients with concomitant hypertension and dyslipidemia: The Respond trial. J Clin Pharmacol. 2007;47:1555-1569.
51. Tse HF, Ro YM, Howes L, et al. Multiple-risk intervention with single-pill amlodipine/atorvastatin therapy helps patients with diverse ethnicity attain recommended therapeutic goals for blood pressure and lipids (the GEMINI-AALA study) [abstract no. PO3-79]. J Hypertens. 2006;24 Suppl 6:329.

52. Flack JM, Victor R, Watson K, et al. Amlodipine/atorvastatin single pill improves goal attainment in the treatment of hypertension and dyslipidemia in African Americans: the CAPABLE trial [abstract]. J Clin Hypertens. 2006;8(6):456.

53. Patel BV, Leslie RS, Foody JM, et al. Adherence with single pill amlodipine/atorvastatin vs a two pill regimen. Vasc Health Risk Manag. 2008;4(3):673-681. 